\title{
Efecto de alimento suplementado con una mezcla probiótica sobre los parámetros productivos de Cavia porcellus, cuy.
}

\section{Effect of feed supplemented with a probiotic mixture on the productive parameters of Cavia porcellus, cuy.}

\author{
Vidalina Andía Ayme ${ }^{l}$ y Ana María Ángeles Lazo ${ }^{l}$ \\ ${ }^{1}$ Universidad Nacional Federico Villarreal, Perú.
}

\begin{abstract}
RESUMEN
El objetivo principal fue evaluar el efecto de alimento suplementado con una mezcla probiótica en la mejora de los parámetros productivos del Cavia porcellus, cuy. Se utilizaron 36 gazapos machos destetados a los $14 \pm 2$ días de edad para el cual se empleó el diseño al azar con cuatro tratamientos, tres repeticiones y tres animales por poza. Los promedios fueron sometidos a la prueba de contraste de Tukey. Fueron los tratamientos T0 como control, T1 $50 \mathrm{ml}$, T2 $100 \mathrm{ml} \mathrm{y} \mathrm{T3} 150 \mathrm{ml}$ de la mezcla probiótica respectivamente. Se brindaron los probióticos en forma diaria, mezclando con el alimento balanceado. Los parámetros evaluados fueron ganancia de peso, consumo de alimento en materia seca, conversión alimenticia y rendimiento de carcasa. Los resultados mostraron que la inclusión de una mezcla probiótica de Lactobacillus acidophilus, L. casei y Saccharomyces cerevisiae en la alimentación del cuy, tuvo efecto significativo $(p<0,05)$ en el consumo de alimento y rendimiento de carcasa. No hubo diferencias significativas para ganancia de peso y conversión alimenticia. Sin embargo, numéricamente en todos los tratamientos con dosis de la mezcla probiótica superó al testigo, además permitió alcanzar el peso comercial del cuy a las ocho semanas.
\end{abstract}

Palabras clave: cavia porcellus, cuyes, probiótico, parámetros productivos.

\begin{abstract}
The main objective was to evaluate the effect of feed supplemented with a probiotic mixture in the improvement of the productive parameters of Cavia porcellus, guinea pig. Thirty-six male kits weaned at $14 \pm 2$ days of age used for which the random design with four treatments, three repetitions and three animals per pool used. The averages subjected to the Tukey contrast test. The treatments were T0 as control, T1 $50 \mathrm{ml}, \mathrm{T} 2100 \mathrm{ml}$ and T3 $150 \mathrm{ml}$ of the probiotic mixture, respectively. Probiotics provided on a daily basis, mixing with the balanced food. The parameters evaluated were weight gain, feed intake in dry matter, feed conversion and carcass performance. The results showed that the inclusion of a probiotic mixture of Lactobacillus acidophilus, L. casei and Saccharomyces cerevisiae in guinea pig, feeding had a significant effect $(p<0.05)$ on
\end{abstract}


feed consumption and carcass performance. There were no significant differences for weight gain and feed conversion. However, numerically in all treatments with doses of the probiotic mixture, it exceeded the control; it also allowed reaching the commercial weight of the guinea pig at eight weeks.

Key words: cavia porcellus, guinea pigs, probiotic, productive parameters.

\section{INTRODUCCIÓN}

$\mathrm{C}$ avia porcellus, cuy, es una especie herbívora originaria de la región andina de Perú, Bolivia, Colombia y Ecuador (Chauca, 1997; Sánchez et al., 2018). Se caracteriza por su fácil manejo, versatilidad alimenticia, bajo costo de producción y por ser una excelente carne con alto contenido nutricional. Constituye fuente de alimentación de la región andina y su demanda está en franco crecimiento en el mercado regional, nacional e internacional por su alto contenido en proteínas, bajo nivel de grasa y el exquisito sabor de su carne (Barboza et al., 2020; León et al., 2016; Cano, 2012; Sánchez et al., 2018). Sin embargo, uno de los mayores problemas sanitarios que afronta la producción y la crianza familiar del cuy, es la presencia de enfermedades infectocontagiosas como la salmonelosis (Aguilar et al., 2011; Cano et al., 2016); la salmonelosis puede causar tasas de mortalidad muy altas superiores al 50\% (Morales, 2017).

Una de las estrategias frecuentes que se emplea para el control de la salmonelosis es la utilización de antibióticos como estrategia de prevención en las granjas de producción de cuyes. No obstante, el uso indiscriminado de los antibióticos tiene consecuencias para la salud pública por la presencia de residuos de antibióticos que puede presentar la carne del cuy. Además, el riesgo de generar resistencia de la salmonella frente al antibiótico es otro de los riesgos para la sanidad animal (Phillips et al., 2004; Cano, 2012). Los problemas asociados al uso de antibióticos para garantizar la producción de animales, permitió la búsqueda de nuevas alternativas más promisorias como el uso de probióticos (Morales, 2017; Cano et al., 2016).

Los probióticos son considerados como microorganismos vivos que confieren efectos beneficiosos en la salud del animal al ser utilizados o administrados en óptimas concentraciones como parte del alimento; una vez ingeridos estos microorganismos pueden modular el equilibrio y las actividades de la microbiota gastrointestinal para garantizar la homeostasis intestinal. No obstante, existen muchos factores y limitaciones tanto en la dieta como en el manejo del probiótico que afectan la microbiota intestinal y que afecta la salud y rendimiento de la producción del animal (Chaucheyras et al., 2010; Markowiak et al., 2018; Cano et al., 2016). Lactobacillus, Streptococcus, Enterococcus, Bacillus y Saccharomyces son los microorganismos probióticos más importantes cuyo efecto beneficioso está asociado a los mecanismos de la exclusión competitiva por el substrato y sitios de adhesión del epitelio intestinal, antagonismos y producción de sustancias antimicrobianas, y la activación del sistema inmunitario del animal (Sun et al., 2020; García et al., 2019; Zihler et al., 2011; Ritchie et al., 2012).

El efecto más significativo de los probióticos es cuando fueron incluidos en la dieta de los animales durante la etapa de destete, inicio de lactancia y cambio en la dieta con forraje (Chaucheyras et al., 2010). Sin embargo, se debe tener en cuenta la cepa o consorcio microbiano utilizado, el proceso metabólico y la supervivencia de los probióticos en el intestino del animal. Asimismo, los microorganismos probióticos en animales monogástricos favorecen la producción de ácido láctico o acético para ayudar a disminuir el $\mathrm{pH}$ intestinal, crear condiciones más favorables para la microbiota residente y disminuir el riesgo de colonización de patógenos. De igual forma, pueden liberar péptidos antimicrobianos como las bacteriocinas que inhiben o controlan la reproducción de bacterias patógenas, o la producción de enzimas capaces de hidrolizar toxinas bacterianas.

Además, pueden estimular o influir en la respuesta inmune (Servin, 2004; Delcenserie et al., 2008; Chaucheyras et al., 2008; Chaucheyras et al., 2010; Markowiak et al., 2018), en consecuencia, junto con el desarrollo intensivo de la crianza de animales, aumentan las expectativas de los productores sobre el uso de probióticos que permiten proteger la salud de las infecciones patógenas y mejorar los parámetros productivos del animal y cada vez más aumentar su demanda en el mercado para la nutrición animal (Markowiak et al., 2018). En este contexto, el objetivo del trabajo fue evaluar el efecto de alimento suplementado con una mezcla probiótica sobre los parámetros productivos del cuy.

\section{MetodologíA}

La investigación se desarrolló en el Laboratorio de Microbiología Industrial y de Alimentos de la Facultad de Ciencias Biológicas y en las instalaciones del Programa de Investigación en Pastos y Ganadería de la Escuela Profesional de Agronomía de la Facultad de Ciencias Agrarias Universidad Nacional de San Cristóbal de Huamanga, Ayacucho.

Para el estudio se emplearon un total de 36 gazapos machos destetados a los $14 \pm 2$ días de edad y con un peso inicial promedio de 250,69 g. La distribución de las unidades experimentales y análisis estadístico se empleó bajo el diseño completamente aleatorio, conformado por cuatro tratamientos con tres repeticiones, con la unidad experimental constituida por tres cuyes por poza. En el presente trabajo se utilizó la metodología de Cano et al., (2016) modificado para las condiciones de Ayacucho.

Los tratamientos aplicados en la suplementación del alimento de los cuyes fueron los siguientes:

- $\quad$ T0 = alimento balanceado

- $\mathrm{T} 1=$ alimento balanceado $+50 \mathrm{ml}$ de mezcla probiótica

- $\mathrm{T} 2=$ alimento balanceado $+100 \mathrm{ml}$ de mezcla probiótica

- $\mathrm{T} 3=$ alimento balanceado $+150 \mathrm{ml}$ de mezcla probiótica 
ISSNe: 2617-9156

A todos los cuyes se les brindó alfalfa (Medicago sativa) como forraje verde al $10 \%$ de su peso vivo, con el objetivo de cubrir la deficiencia de vitamina $\mathrm{C}$.

La mezcla probiótica estuvo formada por 107 células vivas/ml de Lactobacillus acidophilus, Lactobacillus casei y Saccharomyces cerevisiae respectivamente.

Como instalaciones se utilizó un galpón de cuyes de un área de $60 \mathrm{~m} 2$ con techo de calamina transparente y paredes de material noble, se utilizaron doce pozas de malla metálica con marcos de madera, de 0,50 $\mathrm{m}$. de largo, 0,40 $\mathrm{m}$ de ancho y $0,50 \mathrm{~m}$ de altura. Cada una de las pozas contenía tres cuyes y comedero de arcilla con capacidad de 500 gramos para el alimento balanceado y un bebedero de plástico con capacidad de 400 mililitros en la que se colocaba diariamente agua limpia y fresca.

\section{Preparación del alimento balanceado}

El alimento balanceado se formuló en el programa de Pastos y Ganadería de la Universidad Nacional de San Cristóbal de Huamanga y se preparó en forma manual de acuerdo a los porcentajes de ingredientes como se muestra en la tabla 1 .

\section{Selection of the mother plant and selection of the fruit}

The plants with the highest production and those that were disease-free were selected within the plantation. Then the large, healthy and well-matured ears were extracted from the trunk or primary branches since they give uniform and more vigorous seeds.

\section{Tabla 1.}

Composición de alimento balanceado para el cuy

\begin{tabular}{lc}
\hline Ingrediente & $\%$ \\
\hline Pancamel & 31,2 \\
Subproducto de trigo & 19,8 \\
Torta de soya & 19,2 \\
Maíz amarillo & 14,3 \\
Harinilla de trigo & 9,5 \\
Pasta de algodón & 2,9 \\
Carbonato de calcio & 1,3 \\
Grasa de vacuno & 0,9 \\
Harina de pluma & 0,8 \\
Premix & 0,1 \\
\hline Total & 100,0 \\
\hline
\end{tabular}

Ración formulada con $18 \%$ de proteína y 2,7 Mcal $/ \mathrm{Kg}$ de EM

Para la alimentación diaria de los cuyes, el alimento balanceado se brindó en dos tiempos: mañana y tarde, la cantidad de alimento se calculó de acuerdo a edad y peso, además se les brindó una base forrajera - alfalfa verde, equivalente al $10 \%$ del peso vivo.

Para incluir los probióticos en el alimento balanceado, se pesaba el alimento a brindar y se mezclaba en un recipiente de acuerdo a los tratamientos establecidos y se lo colocaba en los comederos. Si había sobrante, se desechaba al día siguiente.

Parámetros evaluados: Consumo de alimento, Ganancia de peso de animal en gramos, Conversión alimenticia y Rendimiento de carcasa.

\section{Análisis de datos}

Para el análisis de los resultados, se usó un diseño completamente al azar que estuvo conformado por cuatro tratamientos con tres repeticiones y tres cuyes como unidad experimental, al cual se realizó el Análisis de Varianza (ANOVA) con un nivel de significancia de 0,05 y para la comparación de los promedios se aplicó la prueba de contraste de Tukey para determinar cuál de los tratamientos era el mejor. Para los análisis estadísticos, se utilizó el software estadístico SAS.

\section{RESUltAdOS Y DISCUSIONES,}

\section{Ganancia de peso}

Los resultados obtenidos en la ganancia de peso indican que no hubo diferencia significativa $(\mathrm{p}>0,05)$ entre los tratamientos al evaluar la mezcla probiótica conformada por Lactobacillus acidophilus, Lactobacillus casei y Saccharomyces cerevisiae. Sin embargo, se demostró numéricamente que mejores tratamientos fueron el T2, T3 y T1 respectivamente, en comparación al T0 (figura 1). 


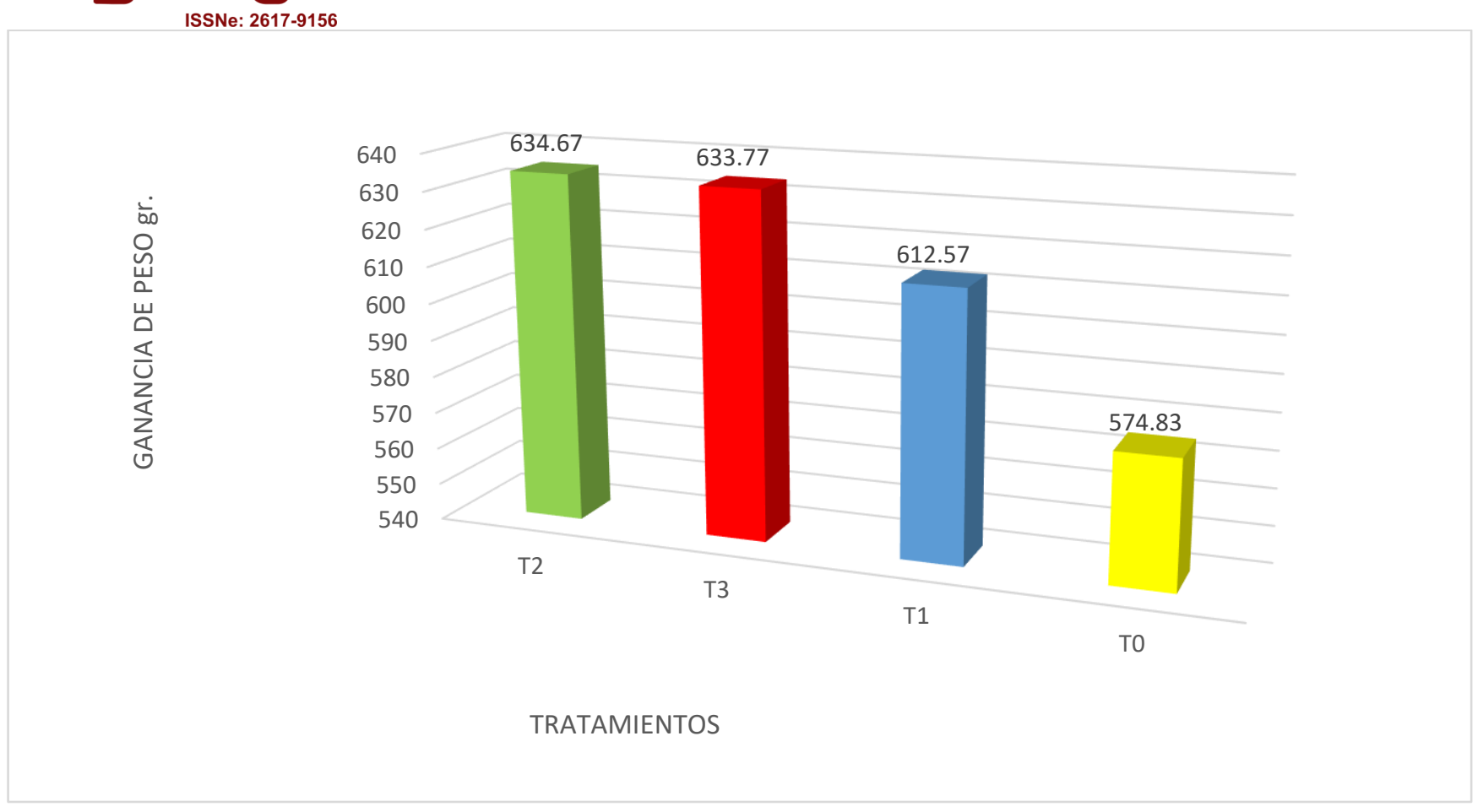

Figura 1. Comparativo del tratamiento sobre la ganancia de peso (g) en cuyes.

\section{Consumo de alimentos}

En el consumo de alimento de los cuyes, se determinó diferencia significativa $(\mathrm{p}>0.05)$ al utilizar la suplementación de una mezcla probiótica en comparación con el testigo. El T3 resultó mejor, seguido del T1 y T2 respectivamente (figura 2 ).

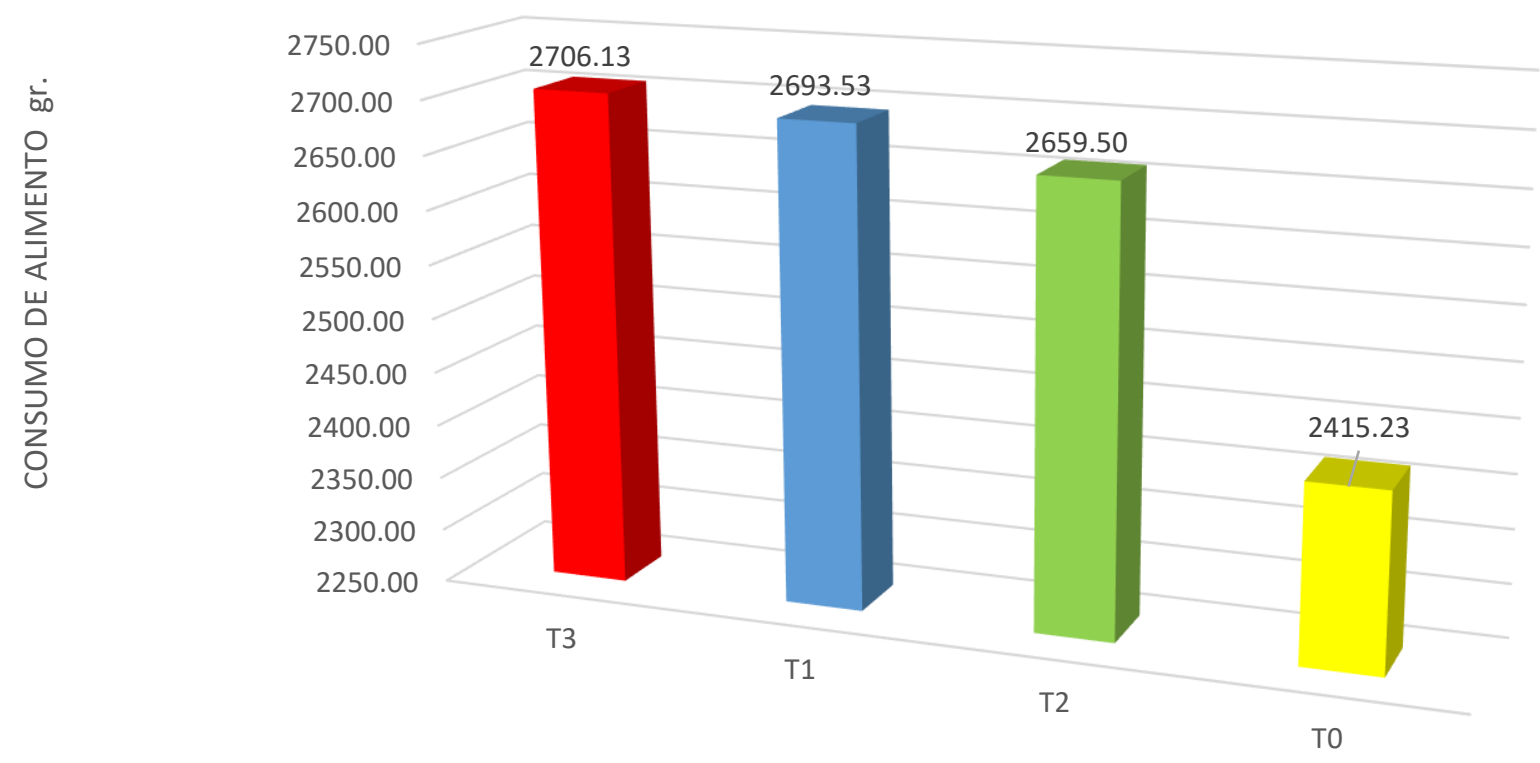

TRATAMIENTOS

Figura 2. Comparación de los promedios de los tratamientos en consumo de alimento.

\section{Conversión alimenticia}

Se demostró que la adición de la mezcla probiótica mejoró la conversión alimenticia promedio de los cuyes, logrando el mejor tratamiento el T2 (figura 3) aunque estadísticamente no hubo diferencia significativa entre los tratamientos con mezcla probiótica y control. 


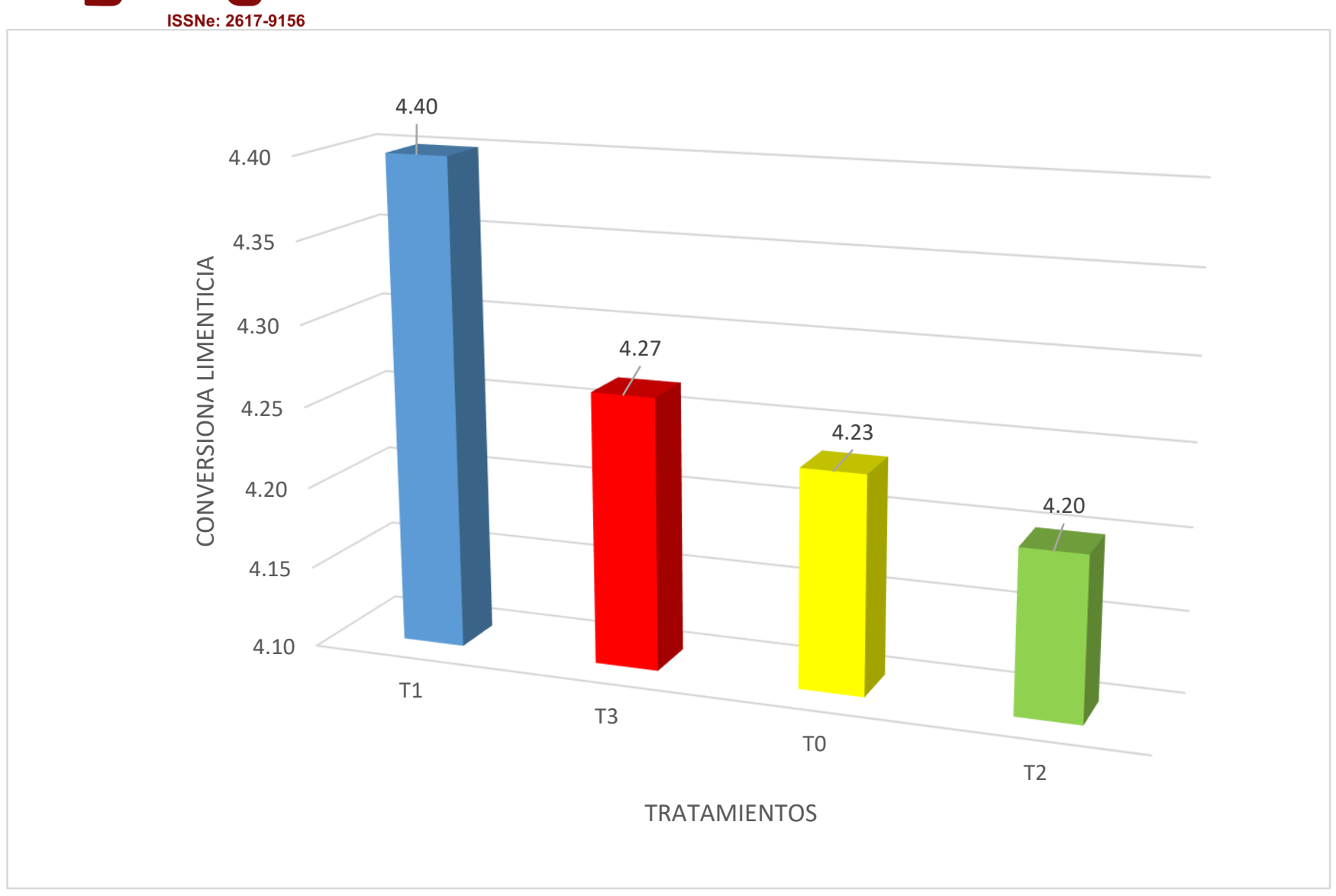

Figura 3. Comparativo de los promedios para conversión alimenticia.

\section{Rendimiento de carcasa}

Se muestra la diferencia significativa $(p>0,05)$ de la mezcla probiótica entre los tratamientos y el control. Se demostró que la mezcla probiótica conformada por
Lactobacillus acidophilus, Lactobacillus casei y Saccharomyces cerevisiae resultó mejor en el T2, T1 y T3 respectivamente y en comparación al T0 (figura 4).

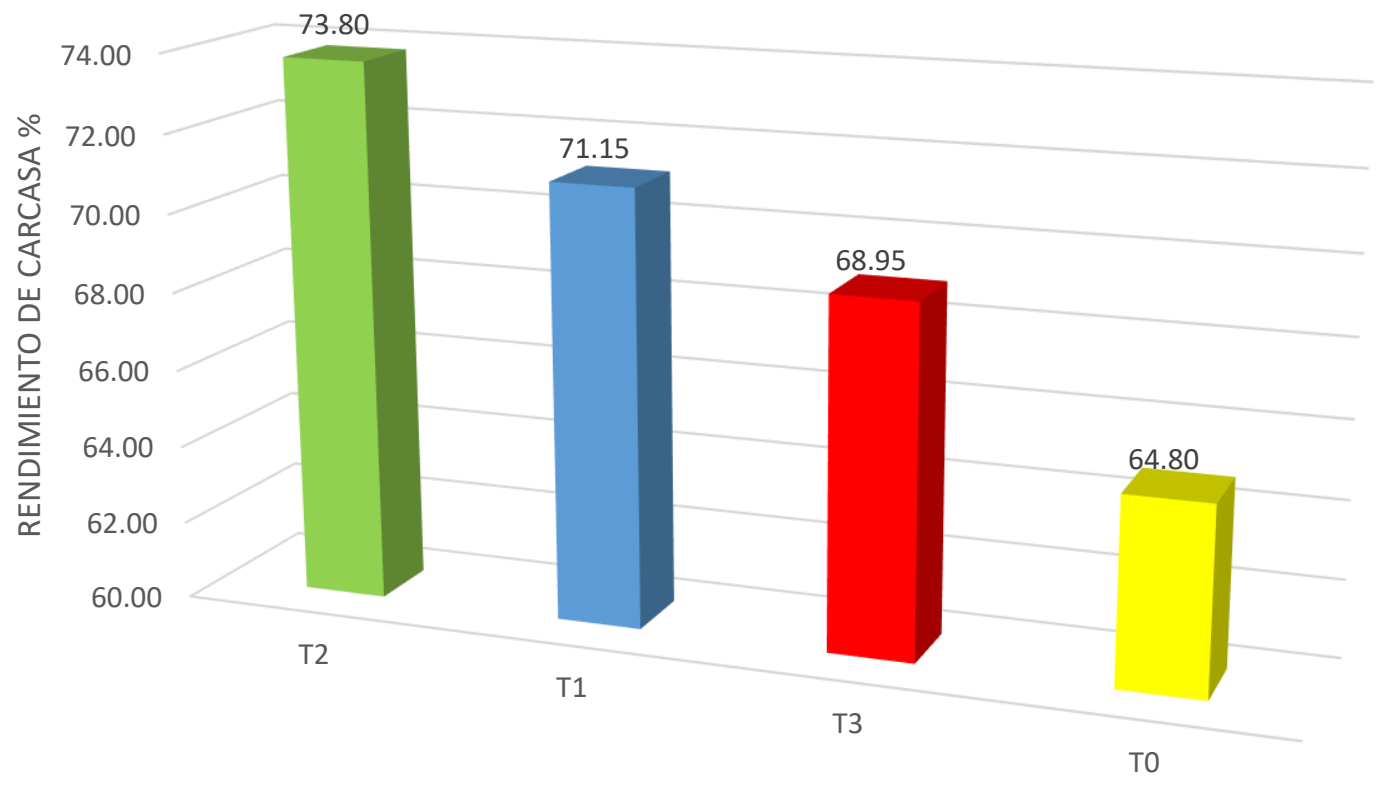

TRATAMIENTOS

Figura 4. Comparativo de los promedios para rendimiento de carcas. 
ISSNe: 2617-9156

\section{DISCUSIÓN}

\section{Ganancia de peso}

Los resultados son coincidentes con los obtenidos por Canto et al., (2019) quienes lograron mayores ganancias de peso del cuy al utilizar alimento suplementado con probiótico. De igual forma, Guevara et al., (2014) lograron numéricamente mayor ganancia de peso del cuy en los tratamientos que contenía Lactobacillus y levadura. Asimismo, Bazay et al., (2014) al utilizar los mananooligosacáridos durante la fase de engorde de los cuyes y, Valdizán (2018) al incluir probiótico, prebiótico y simbiótico en la dieta del cuy encontró una ligera ganancia de peso. Lázaro et al., (2005) encontraron mayor ganancia de peso desde las primeras etapas del crecimiento en marranas al utilizar los probióticos. Molina (2008) con L. acidophilus obtuvo mayor ganancia de peso diaria en comparación al tratamiento control. No obstante, en ninguno de los casos determinaron diferencia significativa entre los tratamientos.

De otro lado, diferentes investigadores lograron diferencia significativa en la ganancia de peso al utilizar probióticos. Jurado et al., (2017) al utilizar alimento balanceado + forraje + L. plantarum en la alimentación del cuy. Cano et al., (2016) al probar en la dieta de los cuyes una mezcla comercial probiótica de Lactobacillus - Bifidobacterium Saccharomyces. Ramírez et al., (2005) en la ganancia del peso vivo promedio de los pollos. Kritas et al., (2008) al utilizar los probióticos en la dieta de los conejos. Flores (2014) al emplear Saccharomyces cerevisiae y Enterococcus faecium en el proceso de engorde de cuyes. De igual forma, Rodríguez (2002) al suplementar Lactobacillus casei, L. acidophilus y Streptococcus faecium en la dieta de pollos, gazapos y lechones. Camacho (2008) al adicionar el probiótico (lacto-sacc) en la dieta de los cuyes. Cano et al., (2016) al incluir una mezcla probiótica en suspensión de Lactobacillus - Bifidobacterium - Saccharomyces en la dieta de los cuyes. Igualmente, Jurgens et al., (1997) al evaluar el suplemento de Saccharomyces cerevisiae durante la fase de crecimiento y desarrollo de los cerdos y, Barros et al., (2008) al agregar probióticos y prebióticos en la dieta de los cerdos determinó que los lechones que recibieron la adición de prebióticos tenían mayor ganancia de peso $(p<0,05)$ en comparación al tratamiento control. No obstante, Torres et al., (2013) al suplementar con un consorcio probiótico en la dieta, reportó que estadísticamente no logró ganancia de peso de los cuyes. El uso de los probióticos en la alimentación animal tiene múltiples ventajas debido a que los microorganismos probióticos actúan sobre el sistema inmunológico de los cuyes para mejorar la ganancia de peso.

\section{Consumo de alimentos}

Los resultados obtenidos coinciden con Flores (2014) quien al utilizar una dieta a base de concentrado y suplementado con probióticos determinó diferencias significativas entre los tratamientos; por tanto, los cuyes que recibieron concentrado suplementado con Saccharomyces cerevisiae y Enterococcus faecium lograron mayor consumo de alimento.

Por otra parte, diferentes investigaciones no encontraron diferencias estadísticas significativas entre los tratamientos, pero sí lograron mayor consumo de alimentos al utilizar probióticos en el crecimiento y desarrollo de los cuyes; Guevara et al., (2014) al utilizar alimento balanceado suplementado con probiótico. Jurado et al., (2017) al evaluar Lactobacillus plantarum en la dieta. Cano et al., (2016) al suplementar una mezcla probiótica comercial conformado por Lactobacillus acidophilus, L. casei, Bifidobacterium longum, Saccharomyces cerevisiae y S. boulardi. Torres et al., (2013) al incluir consorcio probiótico conformado por Enterococcus hirae, L. reuteri, L. frumenti, L. johnsoni y Streptococcus thoraltensis y Bacillus pumilus. Valdizán et al. (2019) al adicionar probiótico, prebiótico y simbiótico en la dieta del cuy.

De igual forma, Lázaro et al., (2005) al añadir a la dieta de lechones, probióticos de Saccharomyces cerevisiae, Bacillus subtillis y Bacillus coagulans. Rodríguez (2002) al incorporar Lactobacillus casei, L. acidophilus y Streptococcus faecium en el alimento de los gazapos y lechones. Barros et al., (2008) al utilizar probióticos en la dieta de la población reproductora de lechones. También, Camacho (2008) al utilizar probiótico en el crecimiento y engorde de cuyes, demostró que todos los tratamientos consumieron igual cantidad de alimento. Sin embargo, Molina (2008) al evaluar el efecto de Lactobacillus acidophilus y Bacillus subtillis como probióticos en el engorde de cuyes, señala que los probióticos a base de B. subtillis no favorecieron el consumo de alimentos.

\section{Conversión alimenticia}

Los resultados obtenidos fueron similares a Molina (2008) quien al utilizar Lactobacillus acidophilus y Bacillus subtillis en la dieta de los cuyes, reportó que la mejor conversión alimenticia logró en el alimento suplementado con los probióticos, aunque no evidenció diferencia significativa entre los tratamientos evaluados.

En tanto, otras investigaciones reportaron que la adición de los probióticos mejora significativamente la conversión alimenticia en los animales, tal como señalan Cano et al., (2016) al evaluar una mezcla probiótica comercial conformada por Lactobacillus - Bifidobacterium Saccharomyces. Ramírez et al., (2005) al utilizar el efecto probiótico del Lactobacillus spp en pollos, Guevara et al., (2014) al determinar el efecto de la suplementación de probióticos (Lactobacillus y levadura) en el crecimiento y engorde los cuyes. Torres et al., (2013) al estimar el efecto de la adición de una cepa probiótica aislada de la microbiota intestinal en el crecimiento del cuy, señalan que el probiótico Lactobacillus contribuye a la absorción más eficiente de nutrientes al degradar macromoléculas para facilitar la difusión por la pared intestinal.

Igualmente, la disponibilidad de compuestos monoméricos como los aminoácidos mejora la eficiencia de utilización de energía logrando incrementar la conversión alimenticia en el cuy. Kritas et al., (2008) al estudiar el efecto de los probióticos en el engorde de los conejos, también evidenciaron mejora en su conversión alimenticia. Flores (2014) al utilizar la adición de Saccharomyces cerevisiae y Enterococcus faecium en la dieta de los cuyes. Camacho (2008) al evaluar el efecto del probiótico (lacto-sacc) en la 
ISSNe: 2617-9156

alimentación de cuyes durante el crecimiento y engorde. Valdizán (2018) al incluir probiótico, prebiótico y simbiótico en la dieta del cuy. Asimismo, Quintana (2009) al suplementar en la dieta de los cuyes harina de cebada más bloque mineral, demostró mayor eficiencia en la conversión alimenticia de los cuyes.

De otro lado, Bazay et al., (2014) al utilizar los mananooligosacáridos procedentes de Saccharomyces cerevisiae durante la fase de engorde de los cuyes, señalan que en ninguno de los tratamientos, estadísticamente, se obtuvieron diferencias significativas en comparación al tratamiento control. Aunque, Jurado et al., (2017) al evaluar Lactobacillus plantarum con características probióticas en los cuyes, mencionan que en algunos tratamientos del forraje $+\mathrm{L}$. plantarum se obtuvo menor conversión alimenticia; por tanto, la conversión alimenticia no fue influenciada por la administración de L. plantarum.

\section{Rendimiento de carcasa}

Al respecto, diferentes investigaciones señalan que los probióticos favorecen mayor rendimiento de carcasa. Sin embargo, estadísticamente no se evidencia diferencia estadística con el tratamiento control. Molina (2008) reportó que la adición L. acidophilus en la dieta de los cuyes facilitó mayor rendimiento de carcasa seguido del tratamiento con B. subtilis. Torres et al., (2013) al realizar su trabajo utilizando un consorcio probiótico aislada de la microbiota intestinal en la suplementación de la dieta del cuy. Lui et al., (2005) al evaluar un consorcio microbiano con Lactobacillus acidophilus, L. casei, Bifidobacterium bifidum, Streptococcus salivarium, Enterococcus faecium, Bacillus subtillis, B. toyoi y Saccharomyces cerevisiae. De igual forma, Bazay et al., (2014) al utilizar los mananooligosacáridos sobre los parámetros productivos de cuyes durante la fase de engorde del cuy señala el rendimiento de carcasa para cada uno de los tratamientos del experimento.

Asimismo, Flores (2014) al utilizar Saccharomyces cerevisiae y Enterococcus faecium durante la fase de crecimiento y engorde de los cuyes determinó un mayor rendimiento de carcasa en comparación al tratamiento con la adición de menor concentración de probiótico y control. Guevara et al., (2014) al determinar la suplementación de probiótico, reportaron que el mejor rendimiento de la carcasa se obtuvo en los cuyes alimentados con alfalfa + concentrado (T1), seguido del uso de levadura y finalmente el tratamiento que contenía tanto Lactobacillus como levadura. Canto et al., (2019) al utilizar alimento suplementado con probiótico, mejoraron el rendimiento de carcasa. No obstante, en ningún caso encontraron diferencias estadísticas entre los tratamientos con probióticos realizados y el control

\section{ConClusión}

La suplementación de una mezcla probiótica de Lactobacillus acidophilus, L. casei y Saccharomyces cerevisiae en la alimentación del cuy tiene efecto significativo $(p>0,05)$ en el consumo de alimento y rendimiento de carcasa; no se encontró diferencia significativa para ganancia de peso y conversión alimenticia.
Sin embargo, numéricamente en todos los tratamientos con dosis de la mezcla probiótica superó al testigo, además permitió alcanzar el peso comercial del cuy a las ocho semanas de crianza.

\section{REFERENCIAS}

[1] Aguilar, G., Bustamante, J., Bazán, V., y Falcón, N. (2011). Diagnóstico situacional de la crianza de cuyes en una zona de Cajamarca. Revista de Investigaciones Veterinarias del Perú, 22 (1), 09-14. Universidad Nacional Mayor de San Marcos.

[2] Barboza F. T., De Souza Rodrigues., S. Leite., C. Gois., H. da Silva Araújo., G. de Alencar y A. Queiroz. (2020). Effect of sex on carcass yield and meat quality of guinea pig. Journal of Food Science and Technology, 1-7. Universidad Federal del Valle de São Francisco. Petrolina, Pernambuco, Brasil.

[3] De Barros, D. S., Júnior, J. G. C., Corrêa, V. S., de Abreu, J. G., Fraga, A. L., Mainardi, F., y Dutra, V. (2008). Efeito da adição de probiótico e prebiótico sobre o ganho de peso, consumo de ração e ocorrência de diarréia em leitões na fase de aleitamento. Revista Brasileira de Saúde e Produção Animal, 9 (3) Universidade Federal da Bahia, Brasil.

[4] Bazay, G., Carcelén, F., Ara, M., Jiménez, R., González, R., y Quevedo, W. (2014). Efecto de los mananooligosacáridos sobre los parámetros productivos de cuyes (Cavia porcellus) durante la fase de engorde. Revista de Investigaciones Veterinarias del Perú, 25 (2), 198-204. Universidad Nacional Mayor de San Marcos.

[5] Camacho, J. (2008). Efecto del probiótico (lacto-sacc) en la alimentación de cobayos criollos machos en la fase de crecimiento y engorde. Tesis de pregrado. Universidad Nacional de Loja, Imbabura. Ecuador. 106 pp.

[6] Cano, J. (2012). Efecto de la suplementación de prebiótico liquido sobre los parámetros productivos en cuyes (Cavia porcellus) durante la fase de crecimiento y engorde. Tesis de pregrado. Universidad Nacional Mayor de San Marcos. 65 pp.

[7] Cano J., F. Carcelén., M. Ara., W. Quevedo., A. Alvarado y R. Jiménez. (2016). Efecto de la suplementación con una mezcla probiótica sobre el comportamiento productivo de cuyes (Cavia Porcellus) durante la fase de crecimiento y acabado. Revista de Investigaciones Veterinarias del Perú, 27 (1), Universidad Nacional Mayor de San Marcos, pp. 51-58.

[8] Canto F., W. Bernal y J. Saucedo. (2019). Efecto de suplementación con probiótico (Lactobacillus) en dietas de alfalfa y concentrado sobre parámetros productivos de cuyes mejorados en crecimiento y engorde. Revista 
ISSNe: 2617-9156

Científica Ciencias Naturales e Ingeniería, 1(2), 39-44.

Universidad Nacional Toribio Rodríguez de Mendoza. Chachapoyas, Amazonas.

[9] Chauca, L. (1997). Producción de Cuyes Cavia porcellus. Food and Agriculture Organization, Naciones Unidas. Roma, 80.

[10] Chaucheyras-Durand F., \& H. Durand. (2010). Probiotics in animal nutrition and health. Beneficial microbes, 1(1), 3-9.

[11] Chaucheyras-Durand F., D Walker \& A. Bach. (2008). Effects of active dry yeasts on the rumen microbial ecosystem: Past, present and future. Animal Feed Science and Technology, 145(1-4), 5-26.

[12] Delcenserie V., D. Martel M. Lamoureux., J. Amiot., Y. Boutin \& D. Roy. (2008). Immunomodulatory effects of probiotics in the intestinal tract. Current issues in molecular biology, 10(1/2), 37 .

[13] Flores,, M. (2014). Efecto de probióticos (Saccharomyces cerevisiae y Enterococcus faecium) en el engorde y sanidad de cuyes-Ayacucho. Tesis de pregrado. Universidad Nacional de San Cristóbal de Huamanga. Ayacucho. 77pp.

[14] Garcia-Mazcorro F., V. Rodriguez-Herrera., G. Marroquín-Cardona \& R. Kawas. (2019). The health enhancer yeast Saccharomyces cerevisiae in two types of commercial products for animal nutrition. Letters in applied microbiology, 68(5), 472-478.

[15] Guevara, J., \& F. Carcelén. (2014). Efecto de la suplementación de probióticos sobre los parámetros productivos de cuyes. Revista Peruana de Química e Ingeniería Química, 17(2), 69-74. Universidad Nacional Mayor de San Marcos.

[16] Jurgens M., A. Rikabi \& D. Zimmerman. (1997). The effect of dietary active dry yeast supplement on performance of sows during gestation-lactation and their pigs. Journal of animal science, 75(3), 593-597.

[17] Jurado H., E. Orbes y L. Mesías. (2017). Evaluation in vivo of Lactobacillus plantarum with probiotic characteristics by blood chemistry, immunohisto química and electron microscopy in Cavia porcellus. Biotecnología en el Sector Agropecuario y Agroindustrial, 15 (2), 11-21. Facultad de Ciencias Pecuarias. Universidad de Nariño, Colombia.

[18] Kritas K., E. Petridou., P. Fortomaris., E. Tzika., G. Arsenos y G. Koptopoulos. (2008). El efecto de los probióticos en la microbiología, la salud y el rendimiento de los conejos de engorde. Asian Australasian Journal of Animal Sciences, 21 (9), 13121317.

Attribution-NonCommercial 4.0 International (CC BY-NC 4.0)
[19] Lázaro D., C. Carcelén., A. Torres \& G. Ara. (2005). Efecto de probióticos en el alimento de marranas sobre los parámetros productivos de lechones. Revista de Investigaciones Veterinarias del Perú, 16 (2), 97-102. Universidad Nacional Mayor de San Marcos.

[20] León Z., E. Silva., A. Wilson y M. Callacna. (2016). Vitamina $\mathrm{C}$ protegida en concentrado de Cavia porcellus, cuy, en etapa de crecimiento - engorde, con exclusión de forraje. Scientia Agropecuaria, 7 (SPE), 259-263. Universidad Nacional de Trujillo, Perú.

[21] Lui F., M. de Oliveira., R. Caires y C. Cancherini. (2005). Desempenho, rendimento de carcaça e pH cecal de coelhos em crescimento alimentados com dietas contendo níveis de probiótico. Ciência Animal Brasileira, 6(2), 87-93. Universidade Estadual Paulista Julio de Mesquita Filho. São Paulo, Brasil.

[22] Markowiak P., y K. Śliżewska. (2018). The role of probiotics, prebiotics and synbiotics in animal nutrition. Gut pathogens, 10 (1), 21.

[23] Molina, M. 2008. Efecto probiótico de Lactobacillus acidophilus y Bacillus subtilis en cuyes (Cavia porcellus) de engorde. Tesis Escuela Politécnica del Ejército. Ecuador. 118 pp.

[24] Morales, M. (2017). Patógenos bacterianos y parasitarios más frecuentes en cuyes de crianza familiarcomercial en tres distritos de la Provincia de Bolognesi, Departamento de Ancash en época de seca. Tesis. Universidad Nacional Mayor de San Marcos. 72 pp.

[25] Phillips I., M. Casewell., T. Cox., B. De Groot., C. Friis., R. Jones y J. Waddell. (2004). Does the use of antibiotics in food animals pose a risk to human health? A critical review of published data. Journal of Antimicrobial Chemotherapy, 53(1), 28-52.

[26] Quintana, E. (2009). Suplementación de dietas a base de alfalfa verde con harina de cebada más una mezcla mineral y su efecto sobre el rendimiento y eficiencia productiva en cuyes en crecimiento en el Valle del Mantaro. Tesis. Universidad Nacional Mayor de San Marcos. 85 pp.

[27] Ramírez R., Z. Santisteban., R. Pérez., R. Valera y M. Medina. (2005). Evaluación del efecto probiótico del Lactobacillus spp de origen aviar en pollitas de inicio reemplazo de la ponedora comercial en los primeros 42 días de edad. REDVET. Revista Electrónica de Veterinaria, 6(9), 1-8. Málaga, España.

[28] Ritchie L \& N. Romanuk. (2012). A meta-analysis of probiotic efficacy for gastrointestinal diseases. PloS one, 7(4).

[29] Rodríguez, L. (2002). Bacterias productoras de ácido 
ISSNe: $2617-9156$

láctico: Efectos sobre el crecimiento y la flora intestinal de pollos, gazapos y lechones. Universidad Complutense de Madrid. 193 pp.

[30] Sánchez-Macías D., L. Barba-Maggi., A. Morales-de la Nuez y J. Palmay-Paredes. (2018). Guinea pig for meat production: A systematic review of factors affecting the production, carcass and meat quality. Meat science, 165-176.

[31] Servin, L. (2004). Antagonistic activities of lactobacilli and bifidobacteria against microbial pathogens. FEMS microbiology reviews, 28(4), 405-440.

[32] Sun Z., T. Wang., N. Demelash., S. Zheng., W. Zhao., X. Chen y G. Qin. (2020). Effect of Yeast Culture (Saccharomyces cerevisiae) on Broilers: A Preliminary Study on the Effective Components of Yeast Culture. Animals, 10(1), 68

[33] Torres C., F. Carcelén., M. Ara., H. San Martín., R. Jiménez., W. Quevedo y J. Rodríguez. (2013). Efecto de la suplementación de una cepa probiótica sobre los parámetros productivos del cuy (Cavia porcellus). Revista de Investigaciones Veterinarias del Perú, 24(4), 433-440. Universidad Nacional Mayor de San Marcos.

[34] Valdizán C., F. Carcelén., M. Ara., S. Bezada., R. Jiménez., A. Asencios y J. Guevara. (2019). Efecto de la inclusión de probiótico, prebiótico y simbiótico en la dieta sobre los parámetros productivos del cuy (Cavia porcellus). Revista de Investigaciones Veterinarias del Perú, 30(2), 590-597. Universidad Nacional Mayor de San Marcos.

[35] Zihler A., C. Chassard., C. Lacroix y M. Gagnon. (2011). Protective effect of probiotics on Salmonella infectivity assessed with combined in vitro gut fermentation-cellular models. BMC microbiology, 11(1), 264 